\title{
Integrated electronic commerce: an international laboratory for business-to- business integration
}

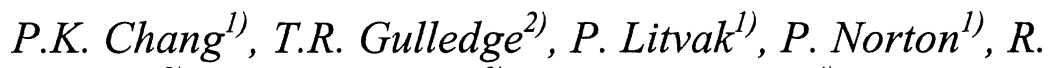
Sommer $^{2)}$, A.J.C. Trappey ${ }^{3)}$, and C. Trappey ${ }^{4)}$

${ }^{1)}$ Oracle Corporation, 1910 Oracle Way, Reston, Virginia 20190 USA

${ }^{2)}$ George Mason University, MS 2E4, Fairfax, Virginia 22030 USA

${ }^{3)}$ National Tsing Hua University, Department of Industrial Engineering, Hsinchu, Taiwan ROC

${ }^{4)}$ National Chiao Tung University, Department of Management Science, Hsinchu, Taiwan ROC

e-mail: pchang@us.oracle.com,gulledge@gmu.edu, plitvak@us.oracle.com,pnorton@us.oracle.com, rsommer@gmu.edu,trappey@nthu.edu.tw, trappey@cc.nctu.edu.tw

\begin{abstract}
Critical issues are emerging in the establishment of virtual environments for international business-to-business e-commerce. One issue is whether all parties (primes and suppliers) can manage extended relationships with information provided by integrated standard software. The issue is more complicated with the inclusion of international trading partners. The joint industry/university laboratory that is described in this paper was designed to explore these complex issues. This paper describes the project, our shared international laboratory, the planned and completed experiments, and interesting results that have been uncovered while executing the project
\end{abstract}

Keywords

Business-to-Business E-Commerce, ERP, Extended Enterprise Integration 


\section{INTRODUCTION}

A critical issue in business-to-business e-commerce is whether all parties (primes and suppliers) can manage extended relationships with information provided by integrated standard software. The issue is more complicated with the inclusion of international trading partners. The joint industry/university laboratory that is described in this paper explores these complex issues. The research effort is initiated by linking four international laboratories via a research center at George Mason University in the USA. Each laboratory is one component in a virtual enterprise that trades and communicates using flexible methodologies that allow for the rapid formation of prime and supplier relationships. The business data are shared via the Internet and processed by Enterprise Resource Planning (ERP) systems at each laboratory. In order to make the experiment meaningful and applicable to the real world business environment, commercial ERP and ecommerce solutions are implemented using heterogeneous systems architectures.

The focus of the virtual laboratory is business-to-business e-commerce, and in particular the suppliers' interface with the prime in the international e-commerce environment. Given the assumption that the prime will be fully integrated (i.e., all transactions will be electronic with full hands-off integration with the prime's ERP system), the suppliers face a number of interface options. These options are a function of the supplier's "business case" for e-commerce. The business case depends on many factors, but typically depends on the number of business transactions processed per time period, the dollar value of the business transactions processed per time period, and the technology sophistication of the supplier. The project focuses on solution approaches and the technology needed for ERP integration between prime and supplier.

This paper describes the laboratory, the planned and completed experiments, and interesting results that have been uncovered while executing the project. The Project was introduced at the 1998 World Congress on Information Technology, and the first phase of the project is planned for full demonstration at the 2000 World Congress on Information Technology. This paper describes results through May 1999. The paper provides a description of all issues associated with implementing the international laboratory, as well as a detailed description of the experiments that have been and will be executed.

The laboratory nodes are defined by the locations of the project's university partners:

- George Mason University, USA

- National Tsing-Hua University, Taiwan

- University of Bremen, BIBA, Germany

- University of Lima, Peru 
- Chung-Ang University, Korea

This paper focuses on the implementation of the USA/Taiwan interface, and their primary industrial partners: Oracle Corporation and Great Plains Software, Inc.

\section{MOTIVATION}

Business transactions among international enterprises trigger other transactions that in turn generate physical, monetary and informational flows across the global supply chain. Supply chains are being engineered to accommodate the growing interdependence among organizations, to improve the competitive position of the participants, to provide better customer service, and to improve the costeffectiveness of firms (Stevens, Gustin, and Ayers; 1997). Part of the redesign of business networks involves changing the scope and function of transactions so that the exchange of structured data is seamless and the movement of inventory is triggered without human intervention (Venkatraman, 1994). These new approaches to supply chain management, combined with new enabling technologies, necessitate the establishment of a virtual environment for international business-tobusiness electronic commerce that includes Small and Medium Enterprises (SMEs). However, no one has fully tested this vision of the technology enabled global supply chain, defined the potential problems that face primes and SMEs, or provided complete architectures for its implementation.

Researchers in Europe (Hedberg, 1998) report that complex E-Commerce problems can be defined and solutions can be demonstrated by staging experiments. Posed with the problem of creating a business-to-consumer Internet infrastructure for Europe, six sponsoring companies launched "e-Christmas," an experimental shopping site. The goal of e-Christmas was to demonstrate Internet based solutions for a new pan-European sales channel. Because of the e-Christmas experiment, 250,000 individuals visited the site, over 30 retailers displayed merchandise on-line, and over 500 transactions were completed. The experiment created core teams in nine European countries that documented the problems and solutions for the pan-European network. The project presented in this paper is similar in that solutions are tested in the experimental laboratory, and then they will be implemented in an actual supply chain.

\section{The Implementation Scenario}

The basic implementation model is what is known as the electronic commerce aggregator model. Figure 1 shows the three components in the model: the customer, the prime (usually a large supply chain lead), and the supplier. The customer places an order over the Internet with the prime via a shopping basket in a storefront environment. The prime processes the order through internal ERP integration, which in turn triggers orders to suppliers. These transactions are varied, depending upon predefined conditions in the environment. For example, a consumer purchase 
can trigger a direct order to ship the final product from the prime to the consumer or send a request for raw materials from the prime to the supplier.

\section{The EC "Aggregator" Model}

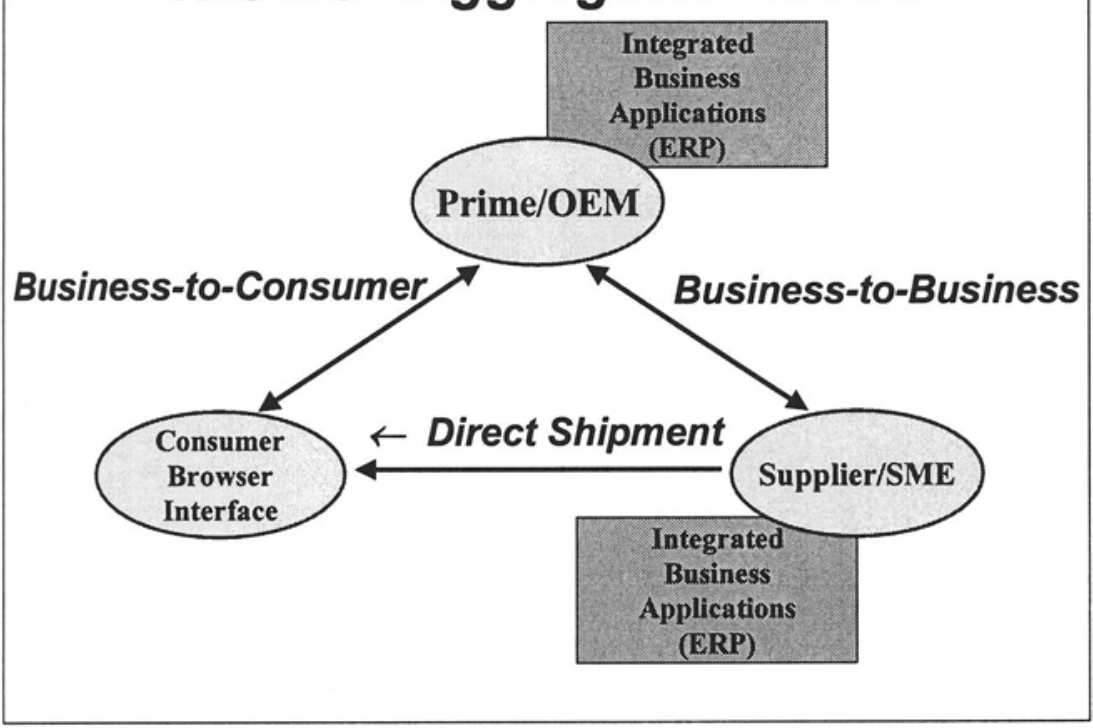

Figure 1. The electronic commerce aggregator model

Finally, there is the shipping and information update problem. Shipments involve movement of the final good to the customer as well as movements of subassemblies, or raw materials from the suppliers to the prime. At each stage of commerce, the internal business systems of the virtual components must be verified and updated.

The full implementation of the aggregator model in our virtual laboratory is indicated in Figure 2. The picture does not indicate the direct shipment linkages, since the focus of our experiments is the business-to-business link.

\section{THE EXPERIMENTS}

This section provides an overview of the experiments that are being executed over the US/Taiwan business-to-business links of figure 2. George Mason University (GMU) plays the role of a major large manufacturer as well as a supplier. National Tsing Hua University (NTHU) plays the role of a supplier. GMU has the following ERP systems installed: Oracle Applications (Release 11) and Great Plains Dynamics $\mathrm{C} / \mathrm{S}+$. NTHU has installed Great Plains Dynamics C/S+. 


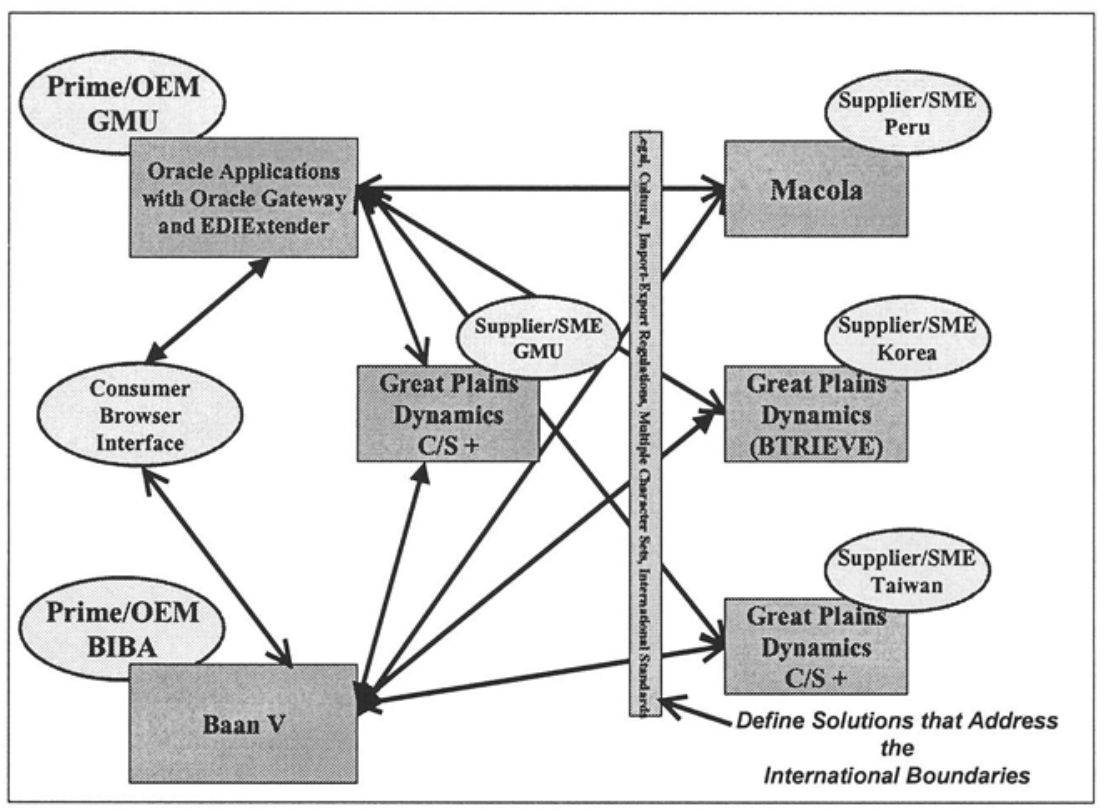

Figure 2. The detailed aggregator model

The experiments are divided into three groups:

- Producer/supplier ERP integration,

- Strategic procurement, and

- Distributed product data management.

This paper reports on the first experiment, which is currently underway.

\section{Producer/Supplier ERP Integration}

This experiment focuses on supply chain integration and management, where integration means automated business transactions based on multiple distributed database updates through ERP and other disparate systems. The automated business transactions are generated directly from the process logic in the suppliers' ERP systems.

The US/Taiwan first phase experiment focuses on the following (discussed in detail below):

- Implement Oracle Applications (R11) at GMU,

- Implement Great Plains Dynamics C/S+ at GMU and NTHU,

- Understand the Oracle/Great Plains Dynamics $\mathrm{C} / \mathrm{S}+$ interface and all specifics of the information exchange that is supported by the ERP process logic,

- Understand the impact of sharing a common set of X12, EDIFACT, or XML implementation conventions among a prime and suppliers (any-to-any data translation), 
- Define the problems in designing, developing, and implementing an interface (i.e., middleware) that meets the requirements of rapidly reconfigurable business-to-business EC between the Oracle and Great Plains solutions,

- Establish the feasibility and defining the requirements for an automated parsing solution that identifies "field-specific" differences among implementation conventions while adapting to information flows that are supported by the ERP process logic,

- Design a middleware solution that meets the requirements,

- Develop a rule-base system for automating the consolidation and database mapping of "field-specific" differences in implementation conventions, and

- Implement an Oracle/Great Plains business-to-business solution.

\section{The Oracle/Great Plains Interface}

What does it mean to understand the Oracle/Great Plains interface? This is a clarification of discussed steps. The problems of establishing connectivity by EDI are understood. The mapping problems are a significant barrier to supplier implementation, because SME cannot justify a business case for VAN-based EDI. However, this is not the focus of this project. This project assumes that firms that have midrange ERP systems have sufficient IS/IT sophistication to overcome this barrier.

This project is focused on rapidly reconfiguring implementation conventions so suppliers can quickly respond to new business relationships from many primes. For example, a trust-based commitment may be rapidly established between a prime and a supplier using browser-based trading process management solutions. Once the relationship us established, the supplier and prime must agree on business rules and the supplier must implement standards in accordance with the requirements of the supplier's ERP systems and the implementation convention that is imposed by the prime. Historically, this process has required time, resources, and technology expertise that are beyond the reach of many suppliers.

The output of the first experiment will be a low-cost "cartridge" (i.e., middleware) that provides easy connectivity between Oracle Applications and Great Plains Dynamics $\mathrm{C} / \mathrm{S}+$. The cartridge considers protocols for data transfer, but more importantly it leverages the process logic within the individual ERP systems. This "cartridge" provides competitive advantage to any large company that is using Oracle Application and doing business with a customer that is using Great Plains Dynamics $\mathrm{C} / \mathrm{S}+$. Over time our plan is to extend this solution to consider other midrange systems and their interaction with Oracle Applications.

\section{CONCLUSIONS}

Most contemporary business-to-business e-commerce solutions have focused on the requirements of large oraganizations. Given the current focus on supplier development, a singular focus on the needs of the supplier is not longer 
appropriate. Solutions are needed that meet the needs of he supplier as well as the prime. Middleware that allows suppliers of easily and affordably do business with users of large standard software solutions (such as Oracle Applications) provides competitive advantage to supplier and prime.

This research project focuses on the needs of the supplier, providing middleware that permits the supplier to do business with the prime in a rapid and affordable fashion. Since ERP systems support different information flows, this is a complex problem, requiring pairwise solutions that focus on specific vendor products. Our international laboratory is designed to provide pairwise middleware for midrange ERP systems that are interacting with Oracle Application. This paper describes the specific aspects of the laboratory that relate to the US/Taiwan implementation. The strategy and detailed steps for providing a solution that addresses the Oracle/Great Plains interface is presented. By its very nature, this research is ongoing. The most recent details of the research effort are posted at http://www.pac.gmu.edu.

\section{REFERENCES}

Hedberg, Sara Reese, "e-Christmas: Seeding Europe's electronic commerce market," IEEE Concurrency, Parallel and Distributed Technology, JanuaryMarch, 1998, Vol 61, pp. 13-15.

Stephens, S., Gustin, C., and Ayers, J, "Reengineering the supply chain - The next hurdle," Information Strategy, The Executive's Journal, Fall, 1997, Vol. 14, No. 1, pp. 13-17.

Venkatraman, N., "IT-enabled business transformation: From automation to business scope redefinition," Sloan Management Review, Winter, 1994, pp. 73-86.

\section{BIOGRAPHIES}

Paul Chang is the Director of Electronic Commerce Solutions for Global Public Sector, Oracle Corporation. Paul had led many large projects and possesses practical experience in Electronic Data Interchange, Electronic Commerce and Enterprise Integration.

Thomas Gulledge is Professor of Public Policy and Engineering at George Mason University and Director of the Policy Analysis Center within the Institute of Public Policy. He lectures in the areas of Organizational Informatics, Electronic Commerce, Planning, Engineering Management, and Enterprise Integration. 
Paul Litvak is the National Director of Logistics \& Electronic Commerce for Oracle Service Industries. He is responsible for developing Supply Chain Management, ERP and Electronic Commerce solutions to support business process modernization for strategic customer accounts in the government, education, health and transportation markets. He is the Oracle technical contact for George Mason University.

Phillip Norton is the Account Manager for Higher Education in the Washington DC Metropolitan area for Oracle Corporation. Phil is responsible for the day to day needs of George Mason University and other institutions of higher education in Washington DC area. He is the main interface between Oracle Corporation and George Mason University.

Rainer Sommer is a member of the George Mason University research faculty. $\mathrm{He}$ is Associate Research Professor of Public Policy and Systems Engineering as well as the assistant director of the Policy Analysis Center of the George Mason University's Institute of Public Policy. Dr. Sommer has consulted and lectured internationally in the field of Enterprise Resource Planning systems, Integrated ECIEDI, software requirements analysis, and Enterprise Integration.

Amy Trappey is Professor of Industrial Engineering and Engineering Management at National Tsing Hua University in Taiwan. Her research interests are in business-to-business electronic commerce, product data management, and manufacturing automation.

Charles Trappey is Professor of Management Science at National Chiao Tung University in Taiwan. His research interests are in business-to-business electronic commerce, international marketing, and consumer behaviour. 